\title{
BMJ Open Gender-stratified analyses of symptoms associated with acute coronary syndrome in telephone triage: a cross- sectional study
}

\author{
Loes T C M Wouters (D) , ${ }^{1}$ Dorien L M Zwart, ${ }^{1}$ Daphne C A Erkelens (D) , \\ Esther De Groot (D) , ${ }^{1}$ Maarten van Smeden, ${ }^{2}$ Arno W Hoes, ${ }^{1}$ \\ Roger A M J Damoiseaux, ${ }^{1}$ Frans H Rutten ${ }^{1}$
}

To cite: Wouters LTCM, Zwart DLM, Erkelens DCA, et al. Gender-stratified analyses of symptoms associated with acute coronary syndrome in telephone triage: a crosssectional study. BMJ Open 2021;11:e042406. doi:10.1136/ bmjopen-2020-042406

- Prepublication history and additional supplemental material for this paper are available online. To view these files, please visit the journal online (http://dx.doi.org/10.1136/ bmjopen-2020-042406).

All authors take responsibility for all aspects of the reliability and freedom from bias of the data presented and their discussed interpretation.

Received 03 July 2020 Accepted 12 May 2021

Check for updates

(c) Author(s) (or their employer(s)) 2021. Re-use permitted under CC BY-NC. No commercial re-use. See rights and permissions. Published by BMJ.

${ }^{1}$ Julius Center for Health Sciences and Primary Care, Universitair Medisch Centrum Utrecht, Utrecht, The Netherlands

${ }^{2}$ Epidemiology, Julius Center for Health Sciences and Primary Care, Utrecht, The Netherlands

Correspondence to

Dr Loes T C M Wouters;

L.T.C.Wouters-2@umcutrecht.n|

\section{ABSTRACT}

Objectives To identify clinical variables that are associated with the diagnosis acute coronary syndrome (ACS) in women and men with chest discomfort who contact out-of-hours primary care (OHS-PC) by telephone, and to explore whether there are indications whether these variables differ among women and men.

Design Cross-sectional study in which we compared patient and call characteristics of triage call recordings between women with and without ACS, and men with and without ACS.

Setting Nine OHS-PC in the Netherlands.

Participants 993 women and 802 men who called OHSPC for acute chest discomfort (pain, pressure, tightness or discomfort) between 2014 and 2016.

Primary outcome measure Diagnosis of ACS retrieved from the patient's medical record in general practice, including hospital specialists' discharge letters.

Results Among 1795 patients (mean age 58.8 (SD 19.5) years, $55.3 \%$ women), $15.0 \%$ of men and $8.6 \%$ of women had an ACS. In both sexes, retrosternal chest pain was associated with ACS (women with ACS vs without $62.3 \%$ vs $40.3 \%, p=0.002$; men with ACS vs without $52.5 \%$ vs $39.7 \%, p=0.032$; gender interaction, $p=0.323$ ), as was pressing/heavy/tightening pain (women $78.6 \%$ vs $61.5 \%, p=0.011$; men $82.1 \%$ vs $57.4 \%, p=<0.001$; gender interaction, $\mathrm{p}=0.368$ ) and radiation to the arm (women $75.6 \%$ vs $45.9 \%, p<0.001$; men $56.0 \%$ vs $34.8 \%$, $\mathrm{p}<0.001$; gender interaction, $\mathrm{p}=0.339$ ). Results indicate that only in women were severe pain $(65.4 \%$ vs $38.1 \%$, $p=0.006$; gender interaction $p=0.007$ ) and radiation to jaw $(50.0 \%$ vs $22.9 \%, p=0.007$; gender interaction $p=0.015)$ associated with ACS.

Ambulances were dispatched equally in women (72.9\%) and men with ACS (70.0\%).

Conclusion Our results indicate there were more similarities than differences in symptoms associated with the diagnosis ACS for women and men. Important exceptions were pain severity and radiation of pain in women. Whether these differences have an impact on predicting ACS needs to be further investigated with multivariable analyses.

Trial registration number NTR7331.

\section{Strengths and limitations of this study}

- We could analyse the very initial conversation with symptom presentation of a large sample of patients calling for acute chest discomfort, without the risk of hindsight bias of the researcher or recall bias of the patient.

- We analysed clinical variables associated with ACS in gender subgroup analyses and across gender with statistical interaction terms.

- Results are generalisable to comparable primary care settings in the UK and Scandinavian countries, and our results may even be generalisable to emergency medical service ('112' or ' 911 ') settings.

- For the purpose of improving telephone triage interviewing, prediction rule development with multivariable regression analysis is needed.

\section{INTRODUCTION}

Adequate triage and early diagnosis is key in patients with acute chest discomfort because they might have an acute coronary syndrome (ACS) for which life-saving early interventions are available. ACS is an umbrella term including ST-elevated myocardial infarction (STEMI), non-ST-elevated myocardial infarction (NSTEMI) and unstable angina pectoris (UAP). ${ }^{1}$ For the diagnosis of ACS, an abnormal electrocardiogram (ST and/ or T-wave abnormalities) and elevated blood levels of troponin I or T are needed. ACS may then be further subdivided into STEMI and NSTEMI if the troponin levels are elevated. ${ }^{1}$ If troponin levels are not elevated (or increased over time), it is called UAP. ${ }^{1}$ Increased preventive measures and development of (timely) effective therapeutic interventions ('time is muscle') have resulted in improved outcomes and prognosis in ACS. ${ }^{2}$ However, telephone triage of patients with chest discomfort, as done in out-of-hours service primary care 
(OHS-PC) and emergency medical services (EMS or ambulance dispatch centres), is challenging because it is difficult to differentiate ACS from other causes of chest discomfort based on symptoms only. ${ }^{34}$ Importantly, the majority of patients with chest discomfort $(80 \%)$ in the Netherlands first approach the general practitioner (GP) or OHS-PC, and $20 \%$ directly call the ambulance (112) or are self-referrals to the emergency department (ED). ${ }^{5}$

Previous hospital-based studies reported a delayed recognition of ACS in women compared with men. ${ }^{67}$ It was suggested that this delayed recognition was related to a less specific presentation in women. ${ }^{89}$ This caused an ongoing debate on whether women with ACS compared with men present with less specific symptoms and how this affects diagnosis, but also treatment, and prognosis. ${ }^{11}$ A recent meta-analysis of 27 studies showed that women with ACS compared with men with ACS had higher odds of presenting with pain between the shoulder blades (OR 2.15, 95\% CI 1.95 to 2.37), nausea or vomiting (OR 1.64, 95\% CI 1.48 to 1.82 ), and shortness of breath (OR 1.34, $95 \%$ CI 1.21 to 1.48 ).${ }^{12}$ Women with ACS had lower odds of sweating (OR $0.84,95 \%$ CI 0.76 to 0.94 ) and presenting with chest pain (OR $0.70,95 \% \mathrm{CI}$ 0.63 to 0.78 ), but in both sexes, chest pain remained the most common symptom (pooled prevalence: men $79 \%$ and women $74 \%) .{ }^{12}$ Importantly, researchers suggested standardisation in methods of symptom assessment is needed because of the difficulties to formulate any definitive statements about symptom presentation, as studies assessed symptoms in different ways (questionnaires or abstracting from medical records). ${ }^{9}{ }^{13-15}$ Abstracting symptom presentation from medical records may dilute symptom presentation, as they are translated by the clinician in medical terminology. ${ }^{16}$ Moreover, many studies suffer from recall or hindsight bias of both patient and researcher as they know the outcome (ACS).

For the clinician or telephone triage nurse, it is crucial to differentiate ACS from other causes of chest discomfort. For that, studies are needed that include female and male patients presenting with chest discomfort, in which women and men who turn out to have ACS are compared with those who do not have ACS. Such studies are scarce. In a study performed among patients with chest discomfort seen at the ED in the USA, 77 women and 244 men with ACS, and 195 women and 240 men without ACS were compared. ${ }^{13}$ Women with ACS more often reported arm pain than women without ACS (47\% vs $32 \%, \mathrm{p}=0.021$ ), while men with ACS reported pressing feeling (63\% vs $54 \%, \mathrm{p}=0.035)$ and chest pain $(72 \%$ vs $60 \%, \mathrm{p}=0.005)$ more often than men without ACS. ${ }^{13}$ In a recent Dutch OHS-PC study among 23 women and 34 men with ACS, and 253 women and 208 men without ACS, symptoms associated with ACS in women and men seemed quite similar and the authors conclude that discriminating ACS in patients with chest discomfort who contacted primary care OHS is difficult in both women and men. ${ }^{17}$

We aimed to identify clinical variables that are associated with the diagnosis ACS in women and men with chest discomfort who contact out-of-hours primary care (OHS-PC) by telephone, and to explore whether there are indications these variables differ among women and men. For analyses, we used the very initial symptom presentation as available from the recorded telephone triage conversations.

\section{METHODS}

We performed a cross-sectional diagnostic factor study with a random sample of 1795 OHS-PC calls for chest discomfort (chest pain, pressure, tightness or discomfort) between 2014 and 2016. ${ }^{18}{ }^{19}$ We first selected calls on the basis of International Code for Primary Care (ICPC, a WHO worldwide code system for primary care) codes (K01, K02, K03, K24, K74, K75, K76, K77, K93, L04, P74 and R02, R98; online supplemental appendix table 1) and keywords thoracic pain, chest pain, myocardial infarction, heart attack and their common abbreviations mentioned by the triage nurse in the electronic medical file (EMF) at the OHS-PC. ${ }^{20}{ }^{21}$ GPs who work at the OHS-PC assign the ICPC codes to the call. We combined ICPC codes and keywords to achieve a sample with a broad variety of symptoms to capture the entire domain of patients suspected of ACS. We listed all available calls of these patients and assigned random numbers with the Random Number Generator function in Microsoft Excel to retrieve a random sample. Calls were excluded before relistening when the patient's age was below 18 years or when the patient did not live in the surrounding area of the OHS-PCs (because then we could not retrieve a diagnosis from the GP of these patients). Calls were excluded during relistening when it not concerned a triage call (eg, intercollegial consultation) or when the recording was of poor quality (figure 1). Adequate methods for sample calculation of a diagnostic factor study is yet lacking. We therefore included a convenient number of patients, that is, at least 80 patients with ACS in each sex category. This number was chosen primarily based on practical and feasibility reasons.

We relistened to the telephone triage recordings to collect information about patient and conversation characteristics, on symptom presentation, medical history, urgency allocation and involvement of a supervising GP in the triage conversation. Gender was considered the selfidentified gender of the patient. Call duration and age were retrieved from the electronic EMF of the OHS-PC. Nine OHS-PCs in the Netherlands participated, serving a total population of 1.5 million people. The diagnosis was made after the phone call, which, in the case of ACS, was nearly always done by the cardiologist $(97.1 \%)$ in the hospital based on (1) symptom presentation, (2) levels of (high-sensitivity) troponin and (3) electrocardiography results. The final diagnoses were provided by the patients' GP, based on the EMF including ED and cardiologist discharge letters, and also the notes from the OHS-PC. We used medical information up to 30 days following the contact with the OHS-PC to allow us to include diagnosis 


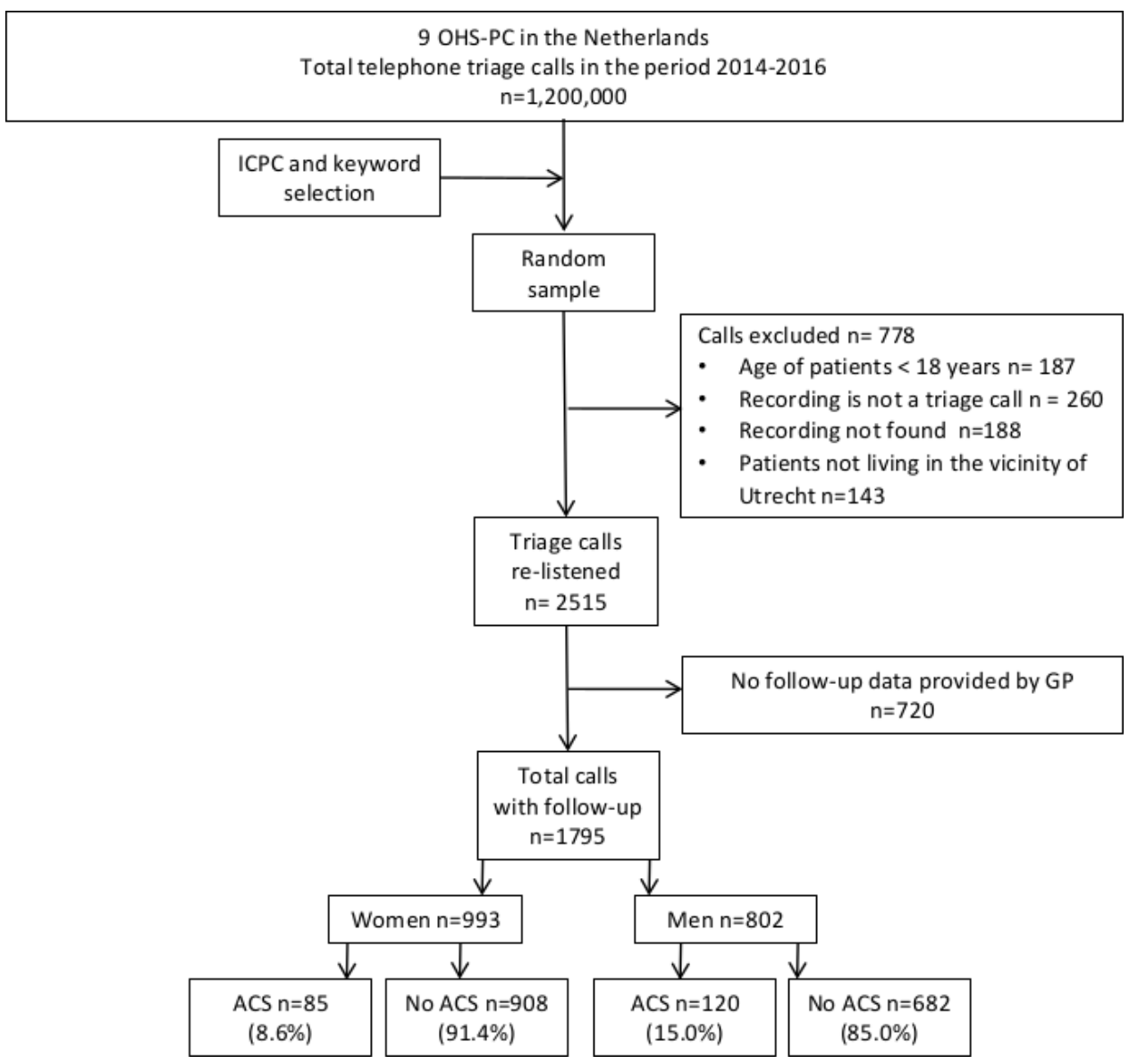

Figure 1 Flowchart study population. ACS, acute coronary syndrome; GP, general practitioner; ICPC, International Code for Primary Care; OHS-PC, out-of-hours primary care.

of ACS that was initially missed because the patient was not referred to the cardiologist on the same day of the OHS-PC contact. In none of the patients in the study we had evidence of a missed diagnosis of ACS.

\section{Context}

In the Netherlands, OHS-PC covers primary care during $73 \%$ of the week hours, and the initial contact is by telephone. In most OHS-PC and EMS, the Netherlands Triage Standard (NTS) is used as a decision support to classify the urgency of the patients' conditions. ${ }^{22}$ Based on the patient's symptom presentation, the triage nurse needs to choose the most appropriate complaint out of 56 'main complaints'. Each NTS main complaint incorporates a decision tree with hierarchically ordered questions, which are similar for men and women. Triage nurses fill out the caller's responses in the semiautomatic NTS system, which then generates urgency allocations linked to a time frame within which the patient should be seen by a physician or ambulance personnel (U0 (reanimation) to U5 (self-care advice), online supplemental appendix table 2). The triage nurse can over-rule this recommendation and upscale or downscale the urgency allocation, often after consulting the supervising GP. ${ }^{23} \mathrm{~A}$ recent validation study showed that the diagnostic accuracy of the NTS for patients with chest discomfort was poor (sensitivity 0.73 (95\% CI 0.68 to 0.78 ) and specificity 0.43 (95\% CI 0.40 to 0.45$)$ ), as calculated on the outcome ACS or other life-threatening events (LTEs). ${ }^{24}$ All telephone calls to the OHS-PC are routinely recorded and archived for 5 years for training and quality control purposes.

\section{Data analyses}

We compared patient and call characteristics between women with and without ACS, and men with and without ACS. For comparison of dichotomous variables, we used the $\chi^{2}$ test or Fisher exact test, and for continuous variables, the independent sample t-test or Mann-Whitney $\mathrm{U}$ test. We performed interaction analysis across gender separately for each clinical variable with logistic regression analyses to explore whether there are indications that these variables are differently associated with the diagnosis ACS among men and women. We analysed the association between urgency allocation and the final diagnosis ACS (alone or including other LTEs) with the $\chi^{2}$ test. We considered pulmonary embolism, thoracic aortic dissection and acute abdominal aneurysm as LTEs; patients with LTEs as well as those with ACS should receive an U1-level urgency.

Data analysis was performed using SPSS, IBM V.25. 


\section{Patient and public involvement}

No patients were involved in setting the research question or the outcome measures, or in developing plans for design; however, they were involved in the implementation of the study. In addition, they were asked to advise on interpretation and writing up of results. Results will be shared and discussed with the national patient community of cardiovascular diseases ('Harteraad').

\section{RESULTS}

Among the 1795 callers with chest discomfort (mean age 58.8 (SD 19.5) years, $55.3 \%$ women), $8.6 \%$ of women and $15.0 \%$ of men had an ACS. In women with an ACS, $18.8 \%$ had a STEMI; $48.2 \%$ an NSTEMI; $20.0 \%$ an UAP; and $13.0 \%$ had non-classified ACS. In men with ACS, $32.5 \%$ had a STEMI; $36.7 \%$ a NSTEMI; $27.5 \%$ an UAP; and $3.3 \%$ non-classified ACS.

A total of $22(2.2 \%)$ women and 23 men $(2.9 \%)$ had another LTE than ACS (eg, pulmonary embolism, thoracic aortic dissection, acute abdominal aneurysm and acute heart failure).

\section{Patient and call characteristics}

Men and women with ACS were older than those without ACS (mean age of women with ACS 73.6 vs without ACS 57.8 years, $\mathrm{p}<0.001$; men 67.2 vs 56.9 years, $\mathrm{p}<0.001$; gender interaction $\mathrm{p}=0.094)$, and the mean duration of the telephone calls was shorter (women 6:47 vs 7:47 min, $\mathrm{p}=0.021$; men $6: 31$ vs $7: 33 \mathrm{~min}, \mathrm{p}=0.004$; gender interaction, $\mathrm{p}=0.803$ ) (table 1 ). The GP was consulted for supervision by the triage nurse in the majority of cases $(52.2 \%$ in women and $55.5 \%$ in men). However, in women with ACS, the GP was less often consulted than in women without ACS ( $41.2 \%$ vs $53.2 \%, p=0.034)$, but in men, such a difference was not observed $(53.3 \%$ vs $55.9 \%, \mathrm{p}=0.607$; gender interaction, $\mathrm{p}=0.208)$.

In around half of the calls, someone else called initially on behalf of the patient $(49.5 \%$ in women vs $54.7 \%$ in men). In cases with ACS, for both sexes, more often someone else called than in those without ACS (in women, $69.4 \%$ vs $47.7 \%$, p $<0.001$; in men, $65.8 \%$ vs $52.8 \%, \mathrm{p}=0.008$; gender interaction $\mathrm{p}=0.251)$. In men with ACS, most often their female partner $(53.3 \%)$ called, while in women with ACS, it was either their male partner $(17.6 \%)$, their daughter $(20.0 \%)$ or a nurse $(17.6 \%)$ (online supplemental appendix table 3 ). Callers expressed concerns in nearly all calls, also in those without an ACS; women with ACS versus women without ACS $97.3 \%$ vs $88.9 \%(p=0.109)$, and men with ACS versus men without ACS $96.3 \%$ vs $86.5 \% \quad(p=0.041)$ (gender interaction, $\mathrm{p}=0.935$ ).

Both women and men with ACS had more often a history of coronary artery disease (women $42.5 \%$ vs $25.1 \%$, $\mathrm{p}=0.017$; and men $57.0 \%$ vs $38.4 \%, \mathrm{p}=0.002$; gender interaction, $\mathrm{p}=0.927$ ), but women with ACS had more often a history of diabetes $(41.4 \%$ vs $14.6 \%, \mathrm{p}<0.001$; gender interaction, $\mathrm{p}=0.079$ ).

\section{Symptom presentation}

Chest pain was the most common complaint, both in patients with and without an ACS (women with ACS and without $98.8 \%$ and $93.1 \%, \mathrm{p}=0.055$; in men $92.4 \%$ and $94.5 \%, \mathrm{p}=0.364$; gender interaction $\mathrm{p}=0.048)$. Retrosternal located chest pain was more common in women and men with ACS than in those without ACS (women $62.3 \%$ vs $40.3 \%, \mathrm{p}=0.002$; and men $52.5 \%$ vs $39.7 \%, \mathrm{p}=0.032$; gender interaction $\mathrm{p}=0.323)$. Also, radiation of pain to the arms was associated with ACS in both sexes (women with vs without ACS $75.6 \%$ vs $45.9 \%, \mathrm{p}<0.001$; and men $56.0 \%$ vs $34.8 \%, \mathrm{p}<0.001$; gender interaction $\mathrm{p}=0.339)$, as was pressing/heavy/tightening chest pain (women with vs without ACS $78.6 \%$ vs $61.5 \%, \mathrm{p}=0.011$; and men $82.1 \%$ vs $57.4 \%, \mathrm{p} \leq 0.001$; gender interaction $\mathrm{p}=0.368)$. Only in women radiation to the jaw had an association with ACS (women $50.0 \%$ vs $22.9 \%, \mathrm{p}=0.007$; men $23.6 \%$ vs $30.4 \%, \mathrm{p}=0.312$; gender interaction, $\mathrm{p}=0.015$ ) and severe pain (eight or more on a Numeric Rating Scale of 0-10) (65.4\% vs $38.1 \%, \mathrm{p}=0.006$; men $2.6 \%$ vs $11.3 \%, \mathrm{p}=0.098$; gender interaction, $\mathrm{p}=0.007)$, which had a differential effect towards the risk of ACS in women. Only in men, stabbing pain was very rare in those with ACS $(8.4 \%$ vs $26.5 \%, \mathrm{p}<0.001)$, but this did not have a differential effect on the diagnosis of ACS between men and women (gender interaction, $\mathrm{p}=0.141$ ).

Of the autonomous nervous system-related symptoms, nausea/vomiting and dizziness/near fainting were not associated with ACS in either sex. A pale or ashen face was associated with ACS in women (55.6\% vs $35.5 \%, \mathrm{p}=0.019$; gender interaction, $\mathrm{p}=0.545)$ and sweating in men ( $52.4 \%$ vs $38.1 \%, \mathrm{p}=0.015$; gender interaction, $\mathrm{p}=0.418$ ), but without a differential effect on the risk of a diagnosis of ACS between women and men. Recognition of symptoms being similar to a previous cardiac event was associated with ACS in men ( $52.9 \%$ vs $32.1 \%, \mathrm{p}=0.004)$ but not clearly for ACS in women $(32.5 \%$ vs $21.4 \%, \mathrm{p}=0.108$; gender interaction, $\mathrm{p}=0.532)$.

Subgroup analyses in 56 women and 58 men with diabetes showed that both women $(85.7 \%$ vs $58.3 \%$, $\mathrm{p}<0.001)$ and men with diabetes $(67.2 \%$ vs $51.5 \%$, $\mathrm{p}=0.033$; gender interaction, $\mathrm{p}=0.119$ ) more often had shortness of breath than those without diabetes, but not as often as chest discomfort (women $90.9 \%$ vs $95.0 \%$, $\mathrm{p}=0.193$; men $89.2 \%$ vs $94.1 \%, \mathrm{p}=0.162$; gender interaction, $\mathrm{p}=0.969)$. Shortness of breath in patients with diabetes was not related to ACS diagnosis (women $81.8 \%$ vs $86.7 \%, \mathrm{p}=0.680$; men $75.0 \%$ vs $66.0 \%, \mathrm{p}=0.615$; gender interaction, $\mathrm{p}=0.520)$.

\section{Diagnoses}

Of the 205 patients with an ACS (85 women, 120 men), $55(26.8 \%$ ) patients had a STEMI (women $18.8 \%$, men $32.5 \%), 85(41.5 \%$ ) had an NSTEMI (women $48.2 \%$, men $36.7 \%$ ), 50 (24.4\%) had UAP (women 20.0\%, men $27.5 \%$ ) and $15(7.3 \%)$ unspecified ACS (women $13.0 \%$, men $3.3 \%$ ), the latter also including two sudden cardiac deaths in women and one in men (table 2). In nearly all 


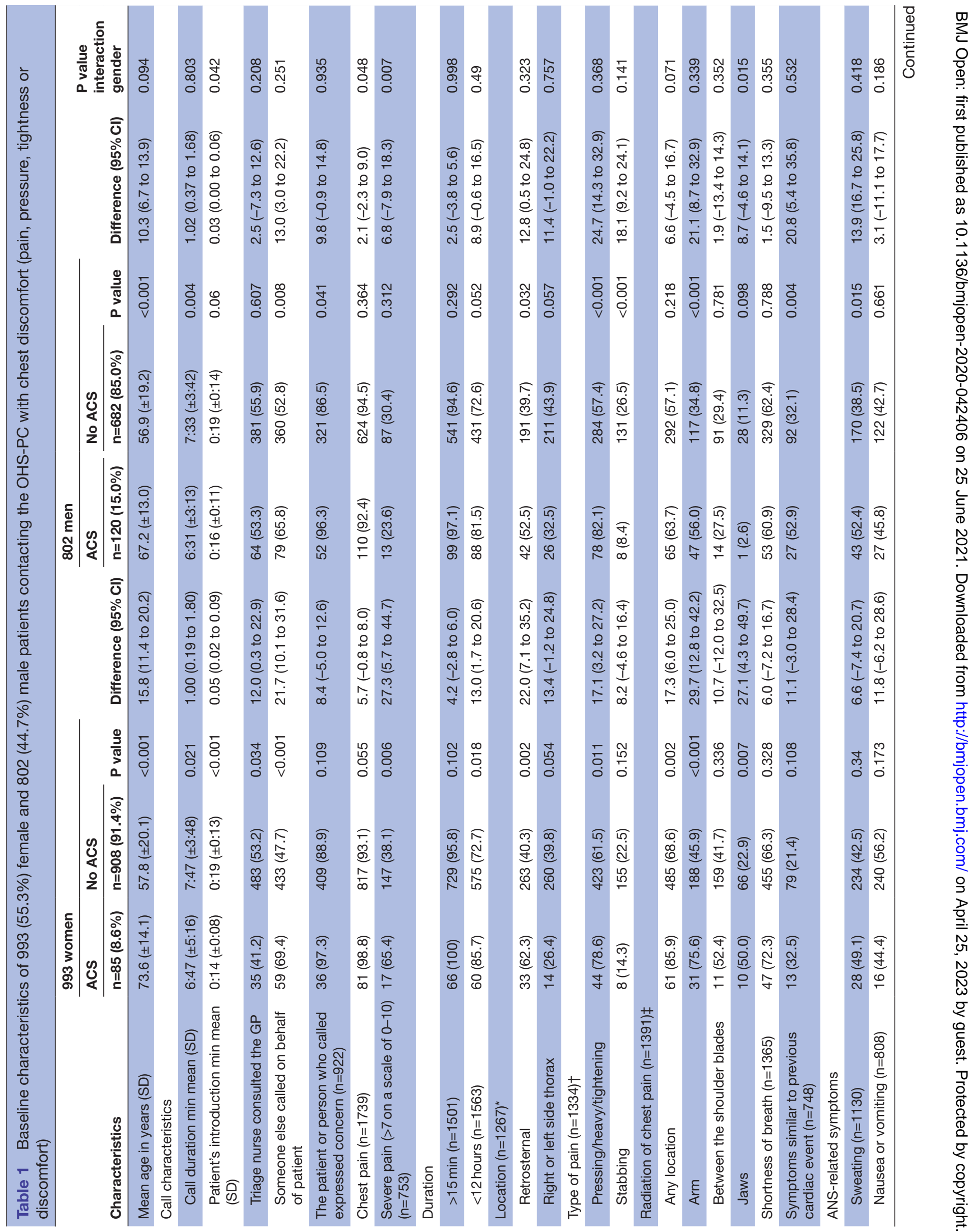




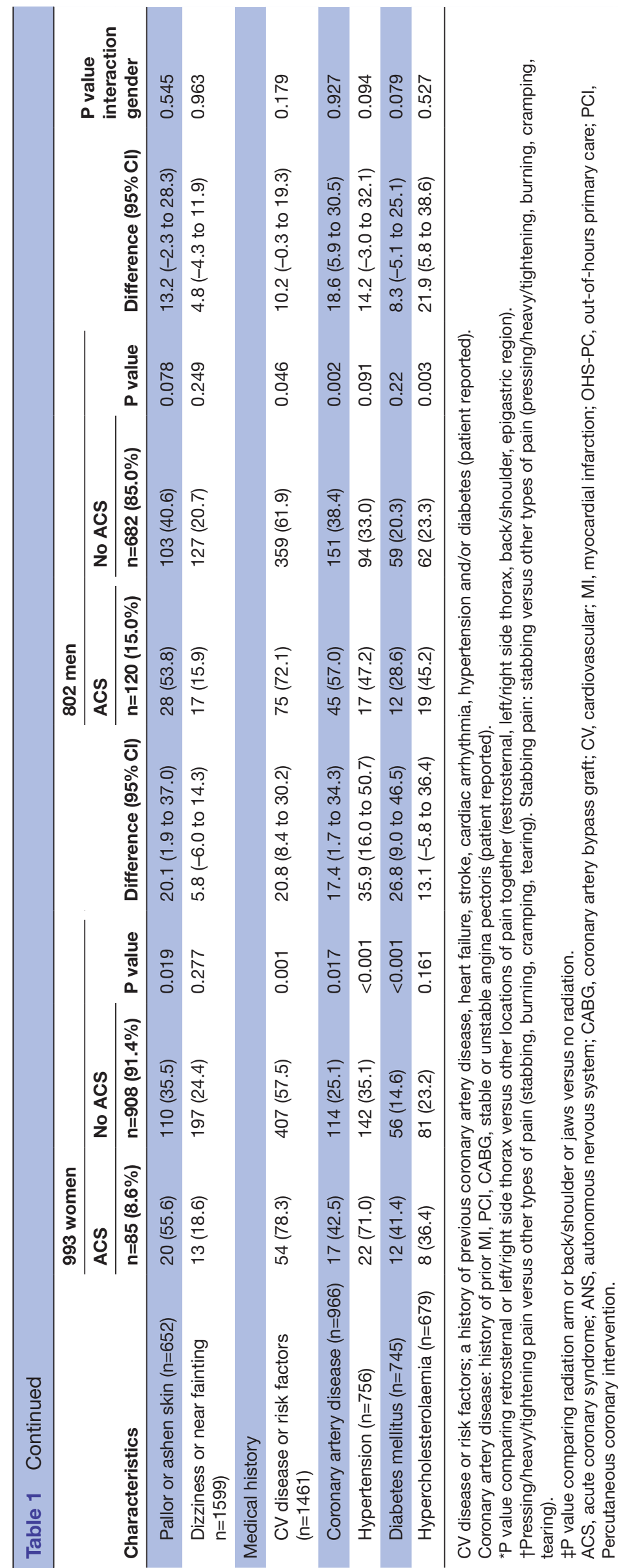


Table 2 Diagnosis of 1795 patients who contacted the out-of-hours primary care for chest discomfort (pain, pressure, tightness or discomfort), divided in women and men

\begin{tabular}{|c|c|c|c|}
\hline & $\begin{array}{l}\text { Women } \\
\mathrm{n}=993(55.3 \%)\end{array}$ & $\begin{array}{l}\text { Men } \\
n=802(44.7 \%)\end{array}$ & $P$ value \\
\hline ACS & $85(8.6)$ & $120(15.0)$ & $<0.001$ \\
\hline ST-elevated myocardial infarction & $16(18.8)$ & 39 (32.5) & 0.037 \\
\hline Non-ST-elevated myocardial infarction & $41(48.2)$ & $44(36.7)$ & 0.114 \\
\hline Unstable angina pectoris & $17(20.0)$ & $33(27.5)$ & 0.250 \\
\hline Non-classified ACS & $11(13.0)$ & $4(3.3)$ & 0.013 \\
\hline Life-threatening events & $22(2.2)$ & $23(2.9)$ & 0.448 \\
\hline Pulmonary embolism & $6(27.3)$ & $7(30.4)$ & 0.815 \\
\hline Thoracic aortic dissection & $4(18.2)$ & $2(8.7)$ & 0.349 \\
\hline Acute abdominal aneurysm & $3(13.6)$ & $2(8.7)$ & 0.598 \\
\hline Other* & $9(40.9)$ & $12(52.2)$ & 0.449 \\
\hline Non-urgent cardiovascular diseases $\dagger$ & $194(19.5)$ & $170(21.2)$ & 0.384 \\
\hline Non-cardiac chest pain, not further specifiedł & $163(16.4)$ & $159(19.8)$ & 0.061 \\
\hline Musculoskeletal pain & $199(20.0)$ & $113(14.1)$ & 0.001 \\
\hline Psychogenic disorders & $139(14.0)$ & $67(8.4)$ & $<0.001$ \\
\hline Gastrointestinal tract disorders & $76(7.7)$ & $62(7.7)$ & 0.951 \\
\hline Respiratory tract disorders & $52(5.2)$ & $45(5.6)$ & 0.727 \\
\hline Other non-urgent diagnoses $\S$ & $63(6.3)$ & $43(5.4)$ & 0.380 \\
\hline
\end{tabular}

*Acute heart failure, stroke, severe chronic obstructive pulmonary disease exacerbation, sepsis, coronary spasm probably caused by hypokalaemia, diabetic ketoacidosis, epileptic insult, bleeding from oesophageal varices, ovarian torsion and ventricular fibrillation. †Stable angina pectoris (including atypical chest pain), stable heart failure, arrhythmias and hypertension. $\ddagger$ Cardiac pathology unlikely after cardiologist's diagnostic work-up, but without differential diagnosis. §Among others: anaemia, malignancy, vasovagal collapse, side effects medication and dermatological diseases. ACS, acute coronary syndrome.

cases $(97.1 \%)$ the ACS diagnosis was made by a cardiologist based on symptom presentation, troponin levels and electrocardiography. Three patients died before arrival of the ambulance (they were classified as acute cardiac death), and one patient died after resuscitation at the ED. Two patients were classified as ACS by the GP; they were not referred to the hospital because of short life expectancy due to cancer.

There were 45 patients with other LTEs $(2.5 \%)$ and the majority of patients had non-urgent medical conditions $(86.1 \%)$. The most common non-urgent diagnoses in both sexes were (1) non-urgent cardiovascular diseases such as stable angina pectoris, stable heart failure and arrhythmias $(19.5 \%$ of all female callers with chest discomfort vs $21.2 \%$ of male callers, $\mathrm{p}=0.384$ ) and (2) non-cardiac unspecified chest pain (women $16.4 \%$ vs $19.8 \%$ men, $\mathrm{p}=0.061$ ). Women more often than men were diagnosed with musculoskeletal problems (women 20.8\% vs men $14.1 \%, \mathrm{p}=0.001$ ) and psychogenic conditions (women $14.0 \%$ vs men $8.4 \%, \mathrm{p}<0.001$ ). Of the patients who were diagnosed with a non-ACS diagnosis, $45.4 \%$ were classified by a cardiologist, $5.5 \%$ were classified by another hospital specialist (eg, pulmonologist or internal medicine specialist) and the remaining patients were diagnosed by a GP.

\section{Urgencies}

Women and men with chest discomfort were equally sent an ambulance $(43.6 \%$ vs $46.6 \%, \mathrm{p}=0.200)$. This was also in women and men who had an ACS $(72.9 \%$ vs $70.0 \%$, $\mathrm{p}=0.647$ ), and in those with either ACS or other LTEs (66.4\% vs $67.1 \%, \mathrm{p}=0.897$ ) (see table 3 ).

\section{DISCUSSION}

For both sexes, retrosternal pain, pain described as pressing, heavy or tightening, and radiation to the arm were associated with ACS in patients who contacted the OHS-PC for chest discomfort. Radiation to the jaw and severe pain were related to ACS in women. Our results indicate there were more similarities than differences in symptoms associated with the diagnosis of ACS for women and men. However, whether these differences have an impact on predicting ACS needs to be further investigated. Women and men with chest discomfort as also those with ACS were equally often sent an ambulance.

Our finding that radiation of pain to the arm and retrosternal ('mid') chest pain were associated with the diagnosis ACS in both sexes was also reported in a study among 2475 patients with acute chest pain in a multicentre ED study. ${ }^{25}$ Another ED study among 1334 patients 
Table 3 Association between urgency allocation, diagnosed ACS and other LTES

\begin{tabular}{|c|c|c|c|c|}
\hline Women & $\begin{array}{l}\text { ACS } \\
n=85(8.6 \%)\end{array}$ & $\begin{array}{l}\text { No ACS } \\
n=908 \\
(91.4 \%)\end{array}$ & $P$ value $1^{*}$ & $\begin{array}{l}P \text { value } \\
2 \dagger\end{array}$ \\
\hline U1 & 62 (72.9) & 371 (40.9) & $<0.001$ & $<0.001$ \\
\hline U2 & $12(14.1)$ & 231 (25.4) & & \\
\hline U3-U5 & $11(13.0)$ & 306 (33.6) & & \\
\hline Men & $\begin{array}{l}\text { ACS } \\
n=120 \\
(15.0 \%)\end{array}$ & $\begin{array}{l}\text { No ACS } \\
n=682 \\
(85.0 \%)\end{array}$ & & \\
\hline U1 & $84(70.0)$ & $290(42.5)$ & $<0.001$ & $<0.001$ \\
\hline U2 & 19 (15.8) & 142 (20.8) & & \\
\hline U3-U5 & 17 (14.2) & $250(36.7)$ & & \\
\hline
\end{tabular}

Women ACS or LTE No ACS or $\mathrm{n}=107 \quad$ LTE (10.8\%) $\quad \mathrm{n}=\mathbf{8 8 6}$

$(89.2 \%)$

\begin{tabular}{|c|c|c|c|c|}
\hline U1 & $71(66.4)$ & 362 (40.9) & $<0.001$ & $<0.001$ \\
\hline U2 & 21 (19.6) & $222(25.1)$ & & \\
\hline U3-U5 & 15 (14.0) & 302 (34.0) & & \\
\hline Men & $\begin{array}{l}\text { ACS or LTE } \\
n=143 \\
(17.8 \%)\end{array}$ & $\begin{array}{l}\text { No ACS or } \\
\text { LTE } \\
\mathrm{n}=659 \\
(82.2 \%)\end{array}$ & & \\
\hline U1 & $96(67.1)$ & 278 (42.2) & $<0.001$ & $<0.001$ \\
\hline U2 & $24(16.8)$ & $137(20.8)$ & & \\
\hline U3-U5 & $23(16.1)$ & 244 (37.0) & & \\
\hline
\end{tabular}

${ }^{*} \mathrm{P}$ value 1: U1 vs U2, U3, U4 and U5.

†P value 2: U1,U2 vs U3,U4,U5.

¥LTEs consist of ACS, pulmonary embolism, thoracic aortic dissection, acute heart failure, stroke, abdominal aortic aneurysm, severe COPD exacerbation, diabetic ketoacidosis, coronary spasm probably caused by hypokalaemia, epileptic insult, bleeding from oesophageal varices, ovarian torsion and ventricular fibrillation. ACS, acute coronary syndrome; LTE, life-threatening event.

with ACS showed that regardless of ethnics status, the most common presenting symptom was retrosternal pain/discomfort of any intensity. ${ }^{26}$ The aforementioned US study in the ED setting reported that radiation to the arm was associated with ACS in women but not for men, and chest pressure was associated with ACS for men but not in women. ${ }^{13}$ The only previously published OHS-PC study reported the opposite; radiation to the arm was associated with ACS in men but not in women. ${ }^{17}$

In our study, women with ACS had more often a history of diabetes and were older than men with ACS, which is in line with other studies. ${ }^{8}{ }^{12}$ Some studies claim that patients with diabetes more often have atypical symptoms of ACS, but a review of eight studies concluded the evidence of these studies was conflicting. ${ }^{27}$ We showed that both women and men with diabetes had more often shortness of breath than those without diabetes, but shortness of breath in patients with diabetes was not associated with ACS. Regarding dispatching the ambulance, the aforementioned OHS-PC study and two EMS studies showed, similar to our findings, that there was no difference in dispatch priorities between men and women with ACS. ${ }^{41728}$ This is in contrast with studies that show delay in hospital presentation of women with ACS. ${ }^{9} 9$

We need to realise that focusing on gender differences may blur the large overlap in symptoms in women and men. Moreover, comparing selectively women with ACS to men with ACS as many previous studies did, is clinically irrelevant. ${ }^{91430}$ Clinicians, including GPs, and triage nurses need to know whether and how women with ACS differ from women without ACS, with the same question for men. Nevertheless, even guidelines stick to comparing those with established disease, and express the view that women with ACS more likely present with less specific symptoms than men with ACS. ${ }^{131}$ Unfortunately, public awareness campaigns follow this reasoning and overemphasise sex differences in women awareness campaigns ('Go Red for Women' in the USA and 'Invisible Me' in Australia). ${ }^{7032}$ An unbalanced attention to symptom differences, while neglecting the much larger overlap, may even introduce new blind spots in recognising ACS in women. ${ }^{33}$

A likely reason behind the predominant message that women present with other ACS symptoms than men is the difference in pathophysiology of coronary artery disease. Women compared with men more often have elongated plaques, located on bifurcations in epicardial coronaries, coronary spasm, microvascular dysfunction and spontaneous coronary dissection. ${ }^{32}{ }^{34}$ These pathophysiological differences have an effect on interventional treatment and prognosis. ${ }^{24}$ However, these differences do not necessarily imply an effect on symptom presentation because the pain pathway is equal in women and men, that is, triggered by myocardial ischaemia. ${ }^{356} \mathrm{~A}$ supply-and-demand mismatch of the myocardial oxygen consumption triggers sensory nerve endings in the myocardium and cause ischaemia symptoms, and this is irrespective of the fact whether the ischaemia is caused by a plaque rupture in an epicardial artery or spasm, or any other cause. ${ }^{36}$ The sex differences in pathophysiology of ACS do therefore not support the belief in differences in ACS symptoms between women and men.

Another reason behind the belief of 'vague' symptom presentation in women with ACS might be that they seem to present a larger number of symptoms than men with ACS, and this may be interpreted as vague by physicians. ${ }^{15} 37$ Presentation of multiple symptoms may influence the prompt recognition of heart disease and initial actions on the part of healthcare providers. ${ }^{37} 38 \mathrm{In}$ a study from 2018, with 2009 women and 976 men hospitalised for myocardial infarction, healthcare providers initially thought symptoms of women $(53.4 \%)$ were less often heart-related than in men $(36.7 \%) .{ }^{37}$ In that study, women and men had the same chest pain symptoms, but women reported more additional symptoms. ${ }^{37}$ In our study, the call duration and the number of GP 
consultations by the triage nurse were similar among women and men, suggesting that triage nurses seem not to experience more difficulties in interpreting symptoms in women than men. This is in line with a prospective study with 2795 patients with chest discomfort in the ED setting that showed the physicians' diagnostic uncertainty for the presence of ACS in women was not more common as compared with men. ${ }^{39}$

Interestingly, in the majority of calls in our study, someone else other than the patient called the OHS-PC (women with ACS $69.4 \%$, without ACS $47.7 \%$; and men with ACS 65.8\%, and without ACS 52.8\%). This was also highlighted in an Australian study among 1681 patients with an acute myocardial infarction (AMI); in $90.5 \%$ of the women with AMI someone else called on behalf of the patient and in $87.8 \%$ of the men with AMI. ${ }^{40}$ According to the protocol in OHS-PC, triage nurses ask the patient to the phone; this is to prevent loss of (paralinguistic) information from the patient him/herself. In our study, in about $50 \%$ of the conversations, the patient took over the phone call.

\section{Strengths and limitations}

A major strength is that we could analyse the very initial conversation with symptom presentation of a large sample of patients calling the OHS-PC because of chest discomfort. We analysed the conversations without knowledge of the eventual diagnosis and have prevented risk of hindsight bias of the researcher or recall bias of the patient. Another strength is that we performed gender subgroup analyses combined with interaction analyses across gender, to investigate whether there are indications that ACS-related symptoms differ among men and women. Furthermore, our results are generalisable to comparable primary care settings, for example, UK and Scandinavian countries, and possibly some other European countries. ${ }^{3}$ Our results may even be generalisable to EMS settings since the prior probability of having an ACS is comparable in EMS setting as in OHS-PC settings. ${ }^{41}$

As the intention of our analysis was to describe whether symptoms were different in patients with ACS from patients without ACS in women and men separately, none of our results can be used to adjust interview questions for the triage nurses. For that purpose, prediction rule development with multivariable analyses is necessary. Also, only with multivariable analysis can it be truly investigated whether the potential differences are clinically relevant in the prediction of ACS. Another limitation is missing values on some clinical variables, a phenomenon common in routine care data, and therefore, the results should be interpreted with caution.

\section{CONCLUSIONS}

Our results indicate there were more similarities than differences in symptoms associated with the diagnosis of ACS for women and men. Important exceptions were pain severity and radiation of pain in women. However, whether these differences have an impact on predicting ACS needs to be further investigated with multivariable analyses.

Acknowledgements The authors thank the out-of-hours primary care foundation Primair Huisartsenposten and all employees of the participating locations for or their cooperation in this study, notably for providing data and technical support.

Contributors FHR and DLMZ are the lead investigators who conceived the research idea and methodology. Funding acquisition was done by FHR, DLMZ and RAMJD. LTCMW and DCE conducted data acquisition. LTCMW performed the analyses and wrote the first draft of the manuscript. She was supervised by FHR and DLMZ, who critically revised the manuscript. DCE, EDG, AWH and RAMJD contributed with and approved the final version of the manuscript. MvS was involved in adjusting the analyses and revising of the manuscript.

Funding This study was funded by an unrestricted grant from (1) the department of general practice of the University Medical Centre Utrecht; (2) a personal promotion grant of $D$ L Zwart, MD, PhD; (3) the foundation the Netherlands Triage Standard; and (4) the foundation 'Stoffels-Hornstra'. It is also part of the IMPRESS study funded by the Dutch Heart Foundation/Dutch Cardiovascular Alliance.

Competing interests None declared.

Patient consent for publication Not required.

Ethics approval The study was approved by the Medical Ethics Committee Utrecht, the Netherlands (reference number WAG/mb/16/003208) and complied with the Declaration of Helsinki. A waiver of informed consent was given because our study had minimal risk to subjects and could otherwise not be carried out logistically. Personal and research data were handled and stored according to the European General Data Protection Regulation.

Provenance and peer review Not commissioned; externally peer reviewed.

Data availability statement Data are available upon reasonable request. The data can be made available for researchers whose proposed use of the data has been approved at the request of the corresponding author, with a signed data access agreement.

Supplemental material This content has been supplied by the author(s). It has not been vetted by BMJ Publishing Group Limited (BMJ) and may not have been peer-reviewed. Any opinions or recommendations discussed are solely those of the author(s) and are not endorsed by BMJ. BMJ disclaims all liability and responsibility arising from any reliance placed on the content. Where the content includes any translated material, BMJ does not warrant the accuracy and reliability of the translations (including but not limited to local regulations, clinical guidelines, terminology, drug names and drug dosages), and is not responsible for any error and/or omissions arising from translation and adaptation or otherwise.

Open access This is an open access article distributed in accordance with the Creative Commons Attribution Non Commercial (CC BY-NC 4.0) license, which permits others to distribute, remix, adapt, build upon this work non-commercially, and license their derivative works on different terms, provided the original work is properly cited, appropriate credit is given, any changes made indicated, and the use is non-commercial. See: http://creativecommons.org/licenses/by-nc/4.0/.

\section{ORCID iDs}

Loes T C M Wouters http://orcid.org/0000-0002-1525-9803

Daphne C A Erkelens http://orcid.org/0000-0002-5846-5201

Esther De Groot http://orcid.org/0000-0003-0388-385X

\section{REFERENCES}

1 Roffi M, Patrono C, et al, Windecker S and Group ESCSD. Esc guidelines for the management of acute coronary syndromes in patients presenting without persistent ST-segment elevation: Task force for the management of acute coronary syndromes in patients presenting without persistent ST-segment elevation of the European Society of cardiology (ESC). Eur Heart J 2015;2016:267-315.

2 Dey S, Flather MD, Devlin G, et al. Sex-Related differences in the presentation, treatment and outcomes among patients with acute coronary syndromes: the global registry of acute coronary events. Heart 2009;95:20-6.

3 Burman RA, Zakariassen E, Hunskaar S. Management of chest pain: a prospective study from Norwegian out-of-hours primary care. BMC Fam Pract 2014;15:51. 
4 Rawshani A, Larsson A, Gelang C, et al. Characteristics and outcome among patients who dial for the EMS due to chest pain. Int $J$ Cardiol 2014;176:859-65.

5 Mol KA, Smoczynska A, Rahel BM, et al. Non-Cardiac chest pain: prognosis and secondary healthcare utilisation. Open Heart 2018;5:e000859.

6 Ting $\mathrm{HH}$, Chen AY, Roe MT, et al. Delay from symptom onset to hospital presentation for patients with non-ST-segment elevation myocardial infarction. Arch Intern Med 2010;170:1834-41.

7 Stehli J, Martin C, Brennan A, et al. Sex differences persist in time to presentation, revascularization, and mortality in myocardial infarction treated with percutaneous coronary intervention. J Am Heart Assoc 2019;8:e012161.

8 Canto JG, Rogers WJ, Goldberg RJ, et al. Association of age and sex with myocardial infarction symptom presentation and in-hospital mortality. JAMA 2012;307:813-22.

9 Chen W, Woods SL, Puntillo KA. Gender differences in symptoms associated with acute myocardial infarction: a review of the research Heart Lung 2005;34:240-7.

10 Canto JG, Goldberg RJ, Hand MM, et al. Symptom presentation of women with acute coronary syndromes: myth vs reality. Arch Intern Med 2007;167:2405-13.

11 Sörensen NA, Neumann JT, Ojeda F, et al. Relations of sex to diagnosis and outcomes in acute coronary syndrome. J Am Heart Assoc 2018;7. doi:10.1161/JAHA.117.007297. [Epub ahead of print: 10 Mar 2018].

12 van Oosterhout REM, de Boer AR, Maas AHEM, et al. Sex differences in symptom presentation in acute coronary syndromes: a systematic review and meta-analysis. J Am Heart Assoc 2020;9:e014733.

13 Devon HA, Rosenfeld A, Steffen AD, et al. Sensitivity, specificity, and sex differences in symptoms reported on the 13-item acute coronary syndrome checklist. J Am Heart Assoc 2014;3:e000586.

14 Araújo C, Laszczyńska O, Viana M, et al. Sex differences in presenting symptoms of acute coronary syndrome: the EPIHeart cohort study. BMJ Open 2018;8:e018798.

15 Canto JG, Canto EA, Goldberg RJ. Time to standardize and broaden the criteria of acute coronary syndrome symptom presentations in women. Can J Cardiol 2014;30:721-8.

16 Ferry AV, Anand A, Strachan FE, et al. Presenting symptoms in men and women diagnosed with myocardial infarction using sex-specific criteria. J Am Heart Assoc 2019;8:e012307.

17 van der Meer MG, Appelman Y, Rutten KHG, et al. Are there gender disparities in symptom presentation or triage of patients with chest discomfort at primary care out-of-hours services? an observational study. BMJ Open 2019;9:e031613.

18 Erkelens DC, Wouters LT, Zwart DL, et al. Optimisation of telephone triage of callers with symptoms suggestive of acute cardiovascular disease in out-of-hours primary care: observational design of the safety first study. BMJ Open 2019;9:e027477.

19 Riley RD, Hayden JA, Steyerberg EW, et al. Prognosis research strategy (progress) 2: prognostic factor research. PLoS Med 2013;10:e1001380.

20 Soler J-K, Okkes I, Wood M, et al. The coming of age of ICPC: celebrating the 21 st birthday of the International classification of primary care. Fam Pract 2008;25:312-7.

21 Olagundoye OA, Malan Z, Mash B, et al. Reliability measurement and ICD-10 validation of ICPC-2 for coding/classification of diagnoses/ health problems in an African primary care setting. Fam Pract 2018;35:406-11.

22 van lerland $Y$, van Veen $M$, Huibers $L$, et al. Validity of telephone and physical triage in emergency care: the Netherlands triage system. Fam Pract 2011;28:334-41.

23 Keizer E, Maassen I, Smits M, et al. Reducing the use of out-of-hours primary care services: a survey among Dutch general practitioners. Eur J Gen Pract 2016;22:189-95.
24 Wouters LT, Rutten FH, Erkelens DC, et al. Accuracy of telephone triage in primary care patients with chest discomfort: a crosssectional study. Open Heart 2020;7:e001376.

25 Rubini Gimenez M, Reiter M, Twerenbold R, et al. Sex-Specific chest pain characteristics in the early diagnosis of acute myocardial infarction. JAMA Intern Med 2014;174:241-9.

26 King-Shier K, Quan H, Kapral MK, et al. Acute coronary syndromes presentations and care outcomes in white, South Asian and Chinese patients: a cohort study. BMJ Open 2019;9:e022479.

27 Stephen SA, Darney BG, Rosenfeld AG. Symptoms of acute coronary syndrome in women with diabetes: an integrative review of the literature. Heart Lung 2008;37:179-89.

28 Herlitz J, Starke M, Hansson E, et al. Characteristics and outcome among women and men transported by ambulance due to symptoms arousing suspicion of acute coronary syndrome. Med Sci Monit 2002;8:CR251-6.

29 Bugiardini R, Ricci B, Cenko E, et al. Delayed care and mortality among women and men with myocardial infarction. J Am Heart Assoc 2017;6.

30 Diercks DB, Owen KP, Kontos MC, et al. Gender differences in time to presentation for myocardial infarction before and after a national women's cardiovascular awareness campaign: a temporal analysis from the can rapid risk stratification of unstable angina patients suppress adverse outcomes with early implementation (crusade) and the National cardiovascular data registry acute coronary treatment and intervention outcomes Network-Get with the guidelines (NCDR action Registry-GWTG). Am Heart $J$ 2010;160:80-7.

31 Mehta LS, Beckie TM, DeVon HA, et al. Acute myocardial infarction in women: a scientific statement from the American heart association. Circulation 2016;133:916-47.

32 Khamis RY, Ammari T, Mikhail GW. Gender differences in coronary heart disease. Heart 2016;102:1142-9.

33 Amalberti R. Navigating safety: necessary compromises and tradeoffs - theory and practice. Heidelberg: Springer, 2013.

34 , Regitz-Zagrosek V, Oertelt-Prigione S, et al, EUGenMed Cardiovascular Clinical Study Group. Gender in cardiovascular diseases: impact on clinical manifestations, management, and outcomes. Eur Heart J 2016;37:24-+.

35 Leach A, Fisher M. Myocardial ischaemia and cardiac pain - a mysterious relationship. Br J Pain 2013;7:23-30.

36 Rosen SD. From heart to brain: the genesis and processing of cardiac pain. Can J Cardiol 2012;28:S7-19.

37 Lichtman JH, Leifheit EC, Safdar B, et al. Sex differences in the presentation and perception of symptoms among young patients with myocardial infarction: evidence from the VIRGO study (variation in recovery: role of gender on outcomes of young AMI patients). Circulation 2018;137:781-90.

38 Goldberg R, Goff D, Cooper L, et al. Age and sex differences in presentation of symptoms among patients with acute coronary disease: the react trial. rapid early action for coronary treatment. Coron Artery Dis 2000;11:399-407.

39 Hillinger P, Twerenbold R, Wildi K, et al. Gender-Specific uncertainties in the diagnosis of acute coronary syndrome. Clin Res Cardiol 2017;106:28-37.

40 Coventry LL, Bremner AP, Jacobs IG, et al. Myocardial infarction: sex differences in symptoms reported to emergency dispatch. Prehosp Emerg Care 2013;17:193-202.

41 Deakin CD, Sherwood DM, Smith A, et al. Does telephone triage of emergency (999) calls using advanced medical priority dispatch (AMPDS) with department of health (DH) call prioritisation effectively identify patients with an acute coronary syndrome? an audit of 42,657 emergency calls to Hampshire ambulance service NHS trust. Emerg Med J 2006;23:232-5. 